PART I

GRAVITATIONAL RADIATION 


\title{
MECHANISMS FOR THE EMISSION AND ABSORBTION OF GRAVITATIONAL RADIATION*
}

\author{
CHARLES W. MISNER \\ Dept. of Physics and Astronomy, University of Maryland, College Park, Md. 20742, U.S.A.
}

\begin{abstract}
Following some introductory comments on the fundamentals or first principles governing jointly the emission and absorption of gravitational waves, a list is given of observational targets or goals for gravitational wave astronomy which have been selected from recent critical reviews. Then theoretical studies of plunge radiation and gravitational synchrotron radiation are surveyed, since in this area new techniques are developing rapidly although new observational prospects have not yet been found.
\end{abstract}

The title of this lecture conveys only a hint of the more precise suggestions of the Organizing Committee. They requested that the talk be "directed towards the future rather than towards reviewing research done in the past" and that it "might contain a synthesis of our theoretical knowledge of the characteristics of the waves bathing the Earth and coming from various sources, so as to provide experimentalists with goals in the design of their future detectors." This is a very demanding assignment, but I can fortunately sidestep the main burden of effort and shorten my own presentation by referring to a number of excellent recent reviews where the desired survey is given more extensively. I will therefore merely state what I take to be the most significant experimental goals, culled from these more detailed surveys, and thereby make room for a few viewpoints which I hope might provoke future theoretical studies in novel directions.

The talk then falls into three parts: first some comments on the fundamentals or first principles governing jointly the emission and absorption of gravitational waves, secondly a list of observational targets or goals for gravitational wave astronomy that I have extracted from recent critical reviews, and thirdly some description of a special area of theoretical studies where new techniques are developing rapidly although they are not currently finding new observational prospects.

\section{Fundamental Interactions Between Gravitational Waves and Other Matter}

We are all familiar from electromagnetic theory with the fact that good emitters are necessarily good absorbers and vice-versa. This requirement on the one hand is necessary to permit thermodynamic equilibrium, and on the other hand follows from the basic microscopic principle of detailed balance. Thus the same fundamentals apply to the study of either emission or detection of gravitational waves, namely the basic interactions between gravitational waves and other fields or matter. It is easiest to begin talking of emitters, but to use linearized theory which is the domain ap-

* Supported in part by NASA grant NGR 21-002-010. 
plicable to all detectors. A familiar focus in this area of theory is the quadrupole radiation formula for the gravitational wave luminosity of a non-relativistic system:

$$
L_{\text {grav. wave }}=\frac{1}{5} \frac{G}{c^{5}}\left\langle\mathscr{I}_{j k} \mathscr{I}_{j k}\right\rangle,
$$

where

$$
\mathscr{I}_{j k}=I_{j k}-\frac{1}{3} \delta_{j k} I_{l l}
$$

is the trace-free part of the moment of inertia tensor $I_{j k}$ of the system, called the 'reduced quadrupole moment' by MTW (Misner, Thorne, and Wheeler, 1973). More easily applied to astrophysical estimates is the restatement of this formula by Dyson (1962) as

$$
L_{\text {grav. wave }} L_{0}=\left(L_{\text {internal }}\right)^{2} .
$$

Here $L_{0}=c^{5} / G=3.6 \times 10^{59} \mathrm{erg} \mathrm{s}^{-1}$ is the natural unit of maximum luminosity, and $L_{\text {internal }} \sim \dddot{\mathscr{I}}_{i j}$ is any asymmetric internal power flow in a system.

Emitters and absorbers which the preceeding formulae suggest are well known, so let me suggest then another viewpoint which may help someone think of still other emitters or absorbers. This viewpoint focusses on anisotropic stresses as the essential feature for interactions with gravitational waves. The linearized field equations for gravitational waves are

$$
\square h_{\mu \nu}=16 \pi T_{\mu \nu}
$$

but the conservation law $T^{\mu v}{ }_{, v}=0$ makes the energy-momentum components redundant, and only the spatial components $\square h_{i j}=16 \pi T_{i j}$ need be studied. Alternatively one notes that it is the space components $h_{i j}$ of the metric perturbation $h_{\mu \nu}=g_{\mu \nu}-\eta_{\mu \nu}$ which contain the transverse traceless basic wave amplitudes $h_{i j}^{T T}$. Thus not only is it sufficient to focus on stresses $T_{i j}$ as sources of gravitational waves, but in fact only the anisotropic or traceless part of the stress contributes as a source of $h_{i j}^{T T}$; simple pressure oscillations are ineffective. For the emission or absorbtion of gravitational waves, then, anisotropic stress is the essential requirement. From this view it is then evident why perfect fluids do not emit, absorb, refract, or reflect gravitational waves. But viscous fluids through viscous shear stress, can absorb gravitational waves (see, e.g. Madore, 1973). Elastic solids in this view are among the most evident candidates for interaction with gravitational waves. With shear stresses $T_{i j}$ found proportional to the wave amplitudes $h_{k l}$ in an elastic solid, one immediately sees that gravitational waves in such a medium move at velocity different from light velocity. They are therefore refracted and partially reflected. But more importantly, energy will be shared between the wave and the solid to make emission and absorbtion possible. Viscosity would then take wave energy absorbed into acoustic oscillations and convert it further into heat.

Unlike the $\mathscr{F}_{j k}$ formula (1), this new centering on anisotropic stresses is not restricted to slowly moving sources, and could therefore lead some insightful person 
to absorbers or emitters more important than those our limited astrophysical imaginations have so far unearthed. One exotic medium which can support shear stresses is an ideal gas of collisionless particles, such as photons or neutrinos in the early radiation dominated universe. Some properties of gravitational waves moving in this medium have been found by Chesters $(1971,1973)$, but they would all be more significant at earlier epochs, of the universe, beyond the range of validity of his methods, so further thought is in order if one is seriously determined to find possible sources of gravitational waves of greater observational significance than those presently imagined.

Another area that could well be studied further is not just Equation (4), but rather the non-linearities that should be included in even the source-free Einstein wave equation. It would be interesting to find ways in which the frequency or pulse shape of a gravitational wave could change, as occurs in non-linear hydrodynamics where a strong sound wave will build up into a shock wave in time. It is perhaps not adequately investigated in general relativity whether a similar phenomena can occur for strong gravitational waves, producing high frequencies out of an initially low frequency wave. This is important since the largest masses are a priori expected to produce very long wavelength gravitational radiation, while shorter wavelengths seem easier to try to observe. The main argument against non-linear frequency multiplication in the Einstein equations is provided by a few examples - the exact solutions for plane and cylindrical gravitational waves. Here, although the full non-linear equations are solved, one basic wave amplitude satisfies a linear equation and no frequency build up is allowed. The needed further studies would then have to consider waves of some more general kind where the Einstein non-linearities are presumably more deep-seated.

We now turn from these few suggestions of where imaginative searches for new sources or detectors might begin, and consider what conclusions have been reached in surveys of now familiar possibilities.

\section{Targets for Technical Achievement in Observing Gravitational Waves}

Five recent surveys of gravitational waves deserve special attention. Sciama (1972) at the Copenhagen relativity conference in 1971 gave an excellent brief summary of the researches directed by Thorne and by Chandrasekhar which have finally settled theoretical discussions on the existence and sign of gravitational radiation damping. He also discussed the Israel-Carter conjecture to the effect that total gravitational collapse always leads to a Kerr metric black hole, that is to a specific and well studied geometry characterized by nothing but the mass and angular momentum of the collapsed system. In Sciama's review one also finds a description of Hawking's arguments leading to upper bounds of $30 \%$ to $50 \%$ on the fraction of the initial rest mass energy which could be converted into gravitational waves in a collision and amalgamation of two black holes. These arguments proceed from various lemmas due to Penrose showing that the area of a black hole (i.e. of an event horizon) cannot decrease. One 
is interested in such exotic processes because only the most extreme conditions conceivable are thought to provide hope for generating gravitational waves at currently detectable intensities.

The Ruffini and Wheeler (1971) article and the Press and Thorne (1972) review were available when Chapters 35-37 of MTW (Misner, Thorne, and Wheeler, 1973) were being written. These chapters provide a textbook introduction and survey of gravitational waves, including possible sources and methods of detection, but Press and Thorne (1971) are more detailed on several points, especially in the analysis of different approaches to detection of gravitational waves. The more recent review Rees gave in Paris at a conference on gravitational waves this June (Rees, 1974) covers the astrophysical aspects of gravitational waves, and goes beyond Press and Thorne in noting a detectability advantage to highly beamed sources even outside our Galaxy, and in considering general energy-type limitations independently of particular source models.

I will not repeat the survey, estimation, and judgement of observability and astrophysical plausibility for all the sources considered in these previous reviews. Rather, I will try to condense and restate some of the conclusions that can be culled from them. But in order to do this I first want to introduce a helpful language. Thus let me define a Gravitational-wave Pulse Unit (GPU) as follows:

$$
1 \mathrm{GPU}=10^{5} \mathrm{erg} \mathrm{cm}^{-2} \mathrm{~Hz}^{-1} \text {. }
$$

This is a measure of the spectral energy density in a gravitational wave pulse at the Earth, so a wave pulse of strength 1 GPU carries $10^{5}$ erg of wave energy across each square centimeter normal to the wavefronts in each $1 \mathrm{~Hz}$ frequency interval at the frequency in question. This kind of unit is appropriate when considering pulses whose frequency spectrum is wider than the bandwidth of the detectors, as is expected for current detectors and likely sources. The energy deposited in such a detector is then obtained by multiplying the pulse intensity $\left(e r g \mathrm{~cm}^{-2} \mathrm{~Hz}^{-1}\right)$ by the integral $\int \sigma(v) \mathrm{d} v$ of the detector cross section over frequency ('resonance integral'). The size of the unit has been chosen to nearly fit a standard (but hypothetical) 1.8 GPU source to which other sources can be conveniently scaled. The reference source in the following general formula is one which is located at the galactic center and isotropically emits one solar rest mass of energy in a gravitational wave pulse of $1 \mathrm{kHz}$ bandwidth:

$$
\begin{aligned}
\text { (pulse intensity) }=1.8 \mathrm{GPU} & \left(\frac{\text { energy in wave }}{M_{\odot} c^{2}}\right) \times \\
& \times\left(\frac{10 \mathrm{kpc}}{\text { distance }}\right)^{2}\left(\frac{10^{3} \mathrm{~Hz}}{\text { bandwidth }}\right)\left(\frac{4 \pi}{\Omega}\right) .
\end{aligned}
$$

Here $\Omega$ is the solid angle into which the source radiates. Using

$$
\left(\int \sigma \mathrm{d} v\right)_{\text {Weber }}=10^{-21} \mathrm{~cm}^{2} \mathrm{~Hz}
$$


one finds that about $400 \mathrm{GPU}$ are required to dump $1 k T$ of energy into a typical Weber bar, and the minimum detectable pulse is determined then by the fraction of $k T$ needed for an acceptable detection efficiency. Rees' (1974) review used the GibbonHawking (1971) estimate which took Weber's limiting sensitivity as about 100 GPU, but Weber (Weber et al., 1973; Lee and Weber, 1974) will suggest substantially lower detectability limits (higher sensitivity) for his present apparatus. Tyson (1973) has used essentially this language in reporting that during a three month period he could exclude the arrival of pulses of strength exceeding $30 \mathrm{GPU}$ which had the frequency $(710 \mathrm{~Hz})$ and other characteristics required by his equipment.

Let me now proceed to use this pulse unit to identify several targets or goals of observational capability which can be related to considerations of astrophysical sources. The reviews I have cited discuss many other sources as well, but I have chosen just a few to serve as benchmarks in the rapidly developing science of gravitational wave astronomy.

\subsection{Current target}

As the current, major, easiest target for a definitive observational consensus I choose the following question: Can one assert the existence or non-existence of pulses of strengths $10^{4} \mathrm{GPU}$ or greater arriving at rates of one per month or more? I believe that all current experiments are designed to provide a clear result at at least this level, but unresolved discrepancies between different laboratories at higher sensitivity levels leave the theorist unsure that the valid scope of any observation is clearly understood. Thus a consensus at this target level is expected only in the nearest future.

This immediate target is already of astrophysical interest, because there is a way of saying that it is not excluded by other observations. One can find a pulse strength and rate at these levels by (i) assuming that the source is at the galactic center (as the nearest very large concentration of somewhat mysterious mass). Then (ii) one considers Sciama's argument (1969, see also Field et al., 1969) that a mass loss rate from the center of the Galaxy exceeding $70 \mathrm{M}_{\odot} \mathrm{yr}^{-1}$ during the past $10^{9} \mathrm{yr}$ would have disturbed stellar orbits in ways contradicted by observation, and therefore chooses this as a limiting average gravitational wave power. But the waves would be easier to detect if they were infrequent but powerful, rather than frequent and feeble, so one opts for a low pulse rate. One pulse per century would not keep one's interest up, however, so (iii) I have chosen one per month as a low rate that an observer could search for. Then each pulse contains $5 M_{\odot} c^{2}$ of gravitational wave energy, and to again maximize our chances of seeing it, we assume (iv) that it is all beamed within $10^{-3} \mathrm{rad}$ of the galactic plane, which is the smallest angle that remains certain to include the Earth. Then with $(\Omega / 4 \pi)=10^{-3}$ and $5 M_{\odot} c^{2}$ energy in Equation (6), and with a bandwidth of $1 \mathrm{kHz}$ as is reasonable for sources in the $\mathrm{kHz}$ band where the present detectors are searching, one finds a pulse strength of $10^{4} \mathrm{GPU}$. This target then imagines a source whose properties are not chosen on grounds of astrophysical plausibility, but are designed to specifications set by our humble ig- 
norance. We admit that Divine ingenuity may surpass human in astrophysical model building, and look for the first observation that could test some of the limits.

\subsection{IMPROVED CURRENT TARGET}

An event rate of one per month but at a higher sensitivity of 10 GPU is not far from the design capabilities of present detectors, and at this level less extreme astrophysical limits characterize the target. One could obtain these pulses from the same mysterious galactic center source as for the first target, but now it would no longer be necessary to postulate any beaming mechanism. But at this level it is not even necessary to assume a source in the Galaxy. Distant galaxies or primordial cosmological chaos (unknown mechanism in both cases) could be the source of these pulses, as the average energy density in a universal flux of $10 \mathrm{GPU}$ pulses at one per month is $10^{-29}$ gm $\mathrm{cm}^{-3}$, that is, just within the cosmological limitations set by the Hubble constant for any form of universal energy density.

\subsection{High RELIABILITY TARgET}

We now turn to consider observational capabilities well beyond those intended in presently operating instruments. A target which asks little more in sensitivity, but a great deal in reliability, is the ability to see one pulse per year of strength $1 \mathrm{GPU}$.

To illustrate the severity of these requirements, consider the following experimental specifications (which are probably not optimal) that give a little better than a $90 \%$ chance of success after 5 yr of running. Let the detection system have $90 \%$ efficiency in finding a gravitational wave pulse of 1 GPU amplitude, and let it report only one false event per decade. Then consider the possible outcomes after it has logged five years of running time, and the significance of each outcome reaching a positive vs a negative conclusion. The 'positive' result finds in favor of the hypothesis that gravitational waves occur with strength $\geqslant 1 \mathrm{GPU}$ at a rate $\geqslant 1$ per year. The 'negative' result favors the hypothesis of no gravitational wave pulses. The experiment can be considered to have failed if it cannot convincingly distinguish these two hypotheses. This failure occurs if the detection system reports a total of either 2 or 3 events in five years, as 2 favors a negative conclusion by odds of only $15: 1$, while 3 favors the positive result by only $5: 1$. But these two awkward outcomes together have less than $10 \%$ probability of occuring under either hypothesis, and all other outcomes are decisive by odds greater than 100:1 (actually $\gtrsim 500: 1$ ), so the experiment will be decisive with greater than $90 \%$ probability.

These high reliability experiments, although they are substantially more difficult than the experiments aimed at the current targets, are of greater astrophysical significance. The source postulated for the 'current targets' was not expected to exist; it simply could not be excluded by other observations or by energy arguments. For this high reliability experiment target, however, there can be proposed a source model for which there are weak astrophysical suggestions. Thus the observational exclusion of this source, by a negative gravitational wave observational finding, would dispose of what is otherwise a significant theoretical possibility. 
The target for high reliability experiments is suggested by the following considerations. Assume there is one star death per year in our Galaxy (estimates run from one to ten). These star deaths could be gravitational collapses which occur without any optical supernova display. It may be that this is the more likely way to produce a black hole, whereas an optical supernova may be associated with the formation of a neutron star as one knows os the case for the crab nebula. The formation of a black hole could release more gravitational wave energy than forming a neutron star, so I have taken an energy relase of $1 M_{\odot} c^{2}$ once a year as conceivable for our Galaxy. This assumes that every case we do not fully understand in the terminal evolution of stars is one where the star converts a substantial fraction of its mass into gravitational waves. Thus a negative observational result at the high reliability target level would show that most star do not end in a blast of gravitational waves, a statement that we cannot make with certainty on other grounds.

\subsection{High SENSITIVITY TARGET}

This next checkpoint for progress in observational capabilities is motivated by an astrophysical source model which is relatively conservative, i.e. quite definitely expected to exist, namely gravitational waves from neutron star formation at the observed optical supernova rate. The target to which this leads is the capability of detecting pulses of strength $10^{-6}$ gravitational-wave pulse unit $\left(10^{-6} \mathrm{GPU}\right)$ occuring at a rate of one per week. The source here would be the Virgo cluster in which there are about 2500 galaxies at distances of $10-15 \mathrm{Mpc}$. For each of these galaxies one may assume a supernova rate of one $\mathrm{SN}$ per $30 \mathrm{yr}$. The further assumption that each optical supernova leads to the formation of a neutron star is fairly plausible but not undebatable. The energy and bandwidth estimates consider that the oscillation which emits gravitational waves is some strong non-linear vibration of the naescent neutron star. The energy then is a substantial fraction of the binding energy of the neutron star; I take $0.05 M_{\odot} c^{2}$. This is not so much energy that the basic structure of the neutron star is altered, so the vibration frequencies will not be very far from the small oscillation frequencies, and on this ground a bandwidth of $100 \mathrm{~Hz}$ is estimated, somewhat narrower than the $10^{3} \mathrm{~Hz}$ used in estimates for total collapse of stars into black holes. But note that an improved knowledge of neutron star structure, whoch one hopes will become available from studies of pulsars and pulsed X-ray sources, will be needed to select the most reasonable detector frequency within the $500-3000 \mathrm{~Hz}$ range to search for these Virgo supernova signals.

The observational targets I have given above are chosen, on the basis of other reviews of a wider range of possibilities, as those most likely to be touched by direct observations along the current line of development. One should also mention, however, possibilities for indirect evidence for the existence of gravitational waves. Later in this symposium Faulkner (1974) will be considering the possibilities for interpreting evolutionary effects in some close binary star systems as governed by gravitational radiation losses. Rees (1974) reviews his suggestion for another indirect effect, namely that primordial gravitational waves with present wavelenths of $1-10 \mathrm{Mpc}$ might lead 
to apparent failures of the virial theorem for small groups (but not rich clusters) of galaxies by increasing the velocity dispersion of the galaxies.

\section{Plunge Radiation and Gravitational Synchrotron Radiation}

We now pass on from theory directed immediately to observational prospects, to some recent specialized developments in theoretical methods which, at present, contribute primarily to our basic understanding of gravitational radiation, and to our physical intuitions concerning it. These involve small masses moving in the fields of larger black holes.

Ruffini and Wheeler (1971) made rough estimates of the energy radiated in gravitational waves when a small mass $\mu$ plunges radially into a black hole of considerably larger mass $M$, and verified that this energy is proportional to $(\mu / M) \mu c^{2}$. The correct coefficient in this formula was then found by Davis et al. (1971), who obtained*

$$
E_{\text {grave. wave }}=0.0104(\mu / M) \mu c^{2}
$$

using fully relativistic methods developed by Regge and Wheeler (1957) and Zerilli (1970). The only approximation required here, beyond the idealization to point particle motion in a Schwarzschild field, is the assumption that $\mu \ll M$. These techniques were then further applied to circular geodesic motion in a Schwarzschild field by Davis et al. (1972) who found (in the limiting case of an $r=6 M$ most tightly bound stable orbit, and to the accuracy I can read their Figure 2) a gravitational wave luminosity of

$$
\begin{aligned}
L_{\text {grav. wave }} & \sim 10^{-3}(\mu / M)^{2} c^{5} / G \sim \\
& \sim 200(\mu / M)^{2}\left[M_{\odot} c^{2} s^{-1}\right] .
\end{aligned}
$$

These two calculations can serve as guides in astrophysical estimates, but confirm most importantly, through the $\mu^{2}$ factor, that small asymmetries are not very effective radiators.

The next set of calculations to be reviewed were part of an attempt (Misner, 1972a) to search for a source model compatible with Weber's (1970) indications of a gravitational wave flux. The aim was to look for particle motions so deep in the gravitational potential well of the black hole that the velocities would be highly relativistic, so the resulting gravitational radiation would have properties analogous to those of electromagnetic synchrotron radiation. These studies did not succeed in producing a plausible model for a source of Weber's gravitational waves, but they did considerably clarify analogies between electromagnetic and gravitational radiation. It now appears that continuing studies may carry the analogy so far that the gravitational theorist may be able to sharpen his intuition by a textbook study of nearly as many model calculations as the electromagnetic theorist has long had available in the standard text chapters on radiation from accelerated charges.

\footnotetext{
* This result has been confirmed by Chung (1973) using entirely independent methods.
} 
The advantages sought in a highly relativistic source were the following. A very large mass would be essential in order to supply the energy to maintain an intense source of gravitational waves at the Galactic center for a substantial period of time such as $10^{8}$ or $10^{9} \mathrm{yr}$. For the efficient production of gravitational waves, strong fields such as those near a black hole appear to be essential. The largest plausible black hole at the center of our own Galaxy would have a mass of the order of $10^{8} M_{\odot}$. The highest frequency for particle motion in such a field is about half a cycle per hour. Gravitational radiation at Weber's kilohertz frequencies would therefore have to be a very high harmonic of order $m \sim 10^{7}$ of the fundamental source frequency, while one knows that highly relativistic charges emit very high harmonics in synchrotron radiation. Another useful feature is that the electromagnetic radiation from a relativistic charge is beamed into a rather narrow angle. We have seen in the discussion of Equation (6) that a beaming factor $\Omega / 4 \pi=10^{-3}$ could be employed, putting all the gravitational radiation from the galactic center into a narrow beam near the galactic equator just wide enough to include the Earth to give a corresponding $10^{3}$ reduction in energy requirements. In the first calculations of geodesic synchrotron radiation (Misner et al., 1972), highly relativistic velocities were simply assumed in an unphysical way. (not derived from gravitational binding energies) to verify that high harmonics and angular beaming could arise in geodesic motion as well as from accelerated motion. These calculations also were simplified by considering radiation of scalar waves rather than tensor (gravitational) waves. The results showed beaming with $\Omega / 4 \pi \sim \gamma^{-1}$ where $\gamma$ is the energy per unit rest mass, as in ordinary synchrotron radiation. The highest harmonics generated, however, were of order $m_{\text {critical }} \propto \gamma^{2}$ in contrast to $m_{\text {critical }} \propto \gamma^{3}$ which holds for accelerated circular orbits.

From this beginning, there were then two lines of development. One pursued the astrophysical prospects for finding a plausible mechanism in which small masses could achieve highly relativistic velocities near black holes so as to get highly beamed, high harmonic, gravitational radiation. This search yielded negative conclusions, and one does not at present see any prospects for finding natural motions which would give rise to such synchrotron-like gravitational radiation. The other line of development was to study in more detail the implications of Einstein's theory for the radiation of gravitational waves by highly relativistic particles.

Let us first consider the search for highly relativistic, astrophysically plausible, sources radiating in synchrotron modes. The first hope was to find GSR (geodesic synchrotron radiation) from stable circular orbits about rapidly rotating black holes, since these orbits have (Boyer-Lindquist) coordinate radii near the horizon and are thus superficially more relativistic than Schwarzschild circular orbits. But already in the first publication (Misner, 1972a) it was known that no GSR is emitted from these orbits. Chrzanowski and Misner had a computation to this effect (reported in Chrzanowski (1972) and Chrzanowski and Misner (1974)), but Bardeen provided the clearer physical explanation, pointing out that the particle motion appeared non-relativistic in a natural (locally non-rotating) reference frame (Bardeen, 1971; Goebel, 1972). For a more complete discussion see Bardeen et al. (1972). 
Plunge radiation in the Kerr metric was then considered. When rotation is high, $a \rightarrow M$, the small mass may obtain relativistic velocities (as measured in the Bardeen locally non-rotating frame) by the time it reaches the neighborhood of the prograde null circular geodesic where synchrotron modes are most easily excited. One finds, however, that essentially all the radiation is beamed down the black hole and does not excape to infinity. This occurs because, even given angular momenta as high as those of a mass in a stable circular orbit, the infall energy is seen primarily as radial momentum in the Bardeen frame, so the radiation beam is directed radially down the black hole. That his result is plausible can be seen from the work of Bardeen et al. (1972), and definitive calculations from the (scalar) wave equation appear in Chrzanowski's thesis (1973).

Could there be some natural source of gravitational synchrotron radiation which we have been too unimaginative to consider? There are two general lines of argument against this, one from polarization, one from the ineffectiveness of even artificial sources when a natural large parameter $(M-a)^{-1}$ is proposed to give large beaming advantages $(\Omega / 4 \pi)^{-1}$. An analysis of the response of Weber type antennas to a polarized source (Tyson and Douglass, 1972; Douglass and Tyson, 1972) shows the reported signals could not come from a strongly polarized source at the Galactic center. But we must postulate that the source mechanism is one which is strongly influenced by the plane of the Galaxy in order to obtain beaming in the required direction. It is then natural to assume that this mechanism is able to strongly influence the plane of polarization of the emitted radiation as well. Misner (Misner, 1972b; Misner et al., 1973) and Hughes (Hughes and Misner, 1973) considered the alternative possibility that magnetic-type components of a black hole gravitational field - curvatures generated by the rotation rather than the mass of the black hole - might give gravitational analogs of Faraday rotations. But they find that these effects only influence radiation emitted toward the poles of the black hole (parallel to its rotation) and vanish for radiation in the equatorial plane. Thus no depolarization from gravitational Faraday rotations is to be expected, and the presumption that any highly beamed source would be strongly polarized is strengthened. On this view, then, the observational reports discourage further speculations of a beamed galactic center source (Misner, 1972b).

Another argument against searching further for a beamed galactic center source somehow tied in to a large black hole is the following. Some large parameter of the black hole should control the beaming factor $\Omega / 4 \pi$. The mass ratio $M / \mu$ could be large, but then linear perturbation theory would apply, and the small mass $\mu$ then influences only the intensity of the radiation, and not its frequency nor angular distribution. The only other elementary large parameter is $[1-(a / M)]^{-1}$. Bardeen (1974) will describe in another paper at this Symposium why this parameter is unlikely to be really large. But in addition, calculations (Chrzanowski and Misner, 1973) show that even the sources thought to be most effective, although unphysical, get turned off rather than enhanced when this parameter is large, although the physical reasons for this behaviour remain unclear. So for these reasons as well, one cannot plausibly look to a near 
critical $(a \rightarrow M)$ rotation velocity of the black hole as the key to synchrotron-like radiation at high harmonics in narrow beams.

Let us now turn to the other line of development from geodesic synchrotron radiation calculations. This is the fuller theoretical understanding of the generation of gravitational waves in new circumstances. Firstly the calculations of synchrotron radiation from energetic $(\gamma \gg 1)$ but unstable circular geodesics were elaborated. Chitre and Price (1972) showed that the techniques of spin weighted spherical harmonics and the Newman-Penrose formalism were more efficient for these calculations than the Regge-Wheeler methods used elsewhere, although they were mistaken in interpreting some results as due to failure of the geometrical optics approximation. More detailed descriptions of the vector (electromagnetic) and tensor (gravitational) geodesic synchrotron radiation are given by Breuer et al. (1972), Breuer et al. (1973), and Breuer and Vishveshwara (1973). These calculations all treated Schwarzschild geometry, and provide details of polarization, spectrum, and angular distributions. The geodesic synchrotron radiation is even more strongly polarized than ordinary synchrotron radiation. The frequency spectrum of radiation from these unstable orbits shows a dependence on the spin of the radiation first noted by Davis et al.(1972) at barely relativistic energies $(\gamma<2.7)$. The high $\gamma$ limit is described by Breuer $e t$ al. (1973) who show that, although scalar or vector geodesic synchrotron radiation has the bulk of the power emitted in a single decade of frequency, the corresponding gravitational (tensor) radiation has approximately equal power emitted in each decade from the fundamental up to the cut-off. Near the equatorial plane, however, the energy per square centimeter available for detection is again found mainly in the highest frequency decade, as the beaming factor $(4 \pi / \Omega)$ is proportional to $\omega^{1 / 2}$.

To exhibit the physical mechanism responsible for the differing geodesic synchrotron spectra in the scalar, vector, and tensor cases required a further development in computational techniques. Chrzanowski and Misner(1974) developed methods of treating vector and tensor wave equations in a high frequency approximation which were little more involved than methods for scalar wave equations, and allowed GSR calculations to be extended to (unstable) relativistic circular geodesics in the Kerr geometry. These methods emphasized the geometric optics approximation rather than separation of variables. A survey of these techniques is given in Misner (1974). This view allows one to see, through a spacetime integral or inner product $\left\langle h_{\mu \nu}{ }^{T T}, T^{\mu \nu}\right\rangle$ a frequency dependent factor arising from the angle between the source momentum and the transverse polarization direction of the emitted radiation. This explains the spin dependence of the spectrum.

The computations (Chrzanowski and Misner, (1974) of geodesic synchrotron radiation from relativistic (unstable) circular orbits in the Kerr metric showed one unexpected feature for which no simple physical explanation has yet appeared. This is the cut-off in radiated entensity as $a \rightarrow M$. Thus for an orbit of fixed high energy $\mu \gamma$, GSR similar to that from unstable Schwarzschild geodesics is emitted, but the total power and the cut-off frequency vary with $a$. Both increase slowly as $a$ increases from zero to $0.95 \mathrm{M}$. But for $a \rightarrow M$, the intensity drops sharply to zero while $\omega_{\text {crit }}$ continues 
to increase slowly. It therefore appears to be exceptionally difficult to excite synchrotron modes in maximally rotating black holes.

These calculations were much simplified by the circumstance that they were limited to high frequencies with $\omega M \gg 1$, so the wavelength is much smaller than the radius $r \sim M$ of a circular geodesic. The radiation then has as its source a 'near zone' which includes only a small neighborhood of the moving mass $\mu$, but excludes the black hole itself. High frequency radiation emission is thus a much more local phenomenon than quadrupole radiation, so the theorist can gain a deeper understanding of the radiation process and finds continuing analogies to electromagnetism. The most succinct statement fo this local view is that of Matzner and Nutku (1973) who have adapted the Weizsäcker-Williams method to computations of the high frequency part of the spectrum of gravitational radiation from highly relativistic particle motions. Since a report of this work will be given at this Symposium (and since I have also reviewed it elsewhere: Misner, 1974), I will not describe it further here.

Although the technical developments described above have not so far borne fruit in leading us to understand probable sources of gravitational wave pulses, they do seem to carry us forward in narrowing the differences between gravity and electromagnetism. They could, therefore, open up the intuitions of physicists and astrophysicists to as yet unexplored source mechanisms, and thus may be an indirect step toward an understanding of observable gravitational radiation.

\section{References}

Bardeen, J. M.: 1971, private communication.

Bardeen, J. M., Press, W. H., and Teukolsky, S. A. : 1972, Astrophys. J. 178, 347.

Bardeen, J. M.: 1974, this volume, p. 132.

Breuer, R. A., Ruffini, R., Tiomno, J., and Vishveshwara, C. V.: 1973, Phys. Rev. D7, 1002.

Breur, R. A., Tiomno, J., and Vishveshwara, C. V.: 1972, Lettere Nuovo Cimento 4, 857.

Breuer, R. A. and Vishveshwara, C. V.: 1973, Phys. Rev. D7, 1008.

Chesters, D.: 1971, Ph.D. Thesis, Univ. of Maryland. See abstract and ordering information in Dissertation Abstracts International 30, 3142-B, 1971

Chesters, D.: 1973, Phys. Rev. D7, 2863.

Chitre, D. M. and Price, R. H.: 1972, Phys. Rev. Letters 29, 185.

Chrzanowski, P. L.: 1972, Bull. Am. Phys. Soc. (II) 17, 472. (Abstract BJ13).

Chrzanowski, P. L.: 1973, Ph.D. Thesis, University of Maryland, unpublished.

Chrzanowski, P. L. and Misner, C. W.: 1974, 'Geodesic Synchrotron Radiation in the Kerr Geometry by the Method of Asymptotically Factorized Green's Functions', University of Maryland, Center for Theoretical Physics Technical Report, to be published.

Chung, K. P.: 1973, Nuovo Cimento 14B, 293.

Davis, M., Ruffini, R., Press, W. H., and Price, R. H.: 1971, Phys. Rev. Letters 27, 1466; see also Davis, M., Ruffini, R., and Tiomno, J.: 1972, Phys. Rev. D5, 2932.

Davis, M., Ruffini, R., Tiomno, J., and Zerilli, F.: 1972, Phys. Rev. Letters 28, 1352.

Douglass, D. H. and Tyson, J. A.: 1972, Astrophys. J. 178, 341.

Dyson, F. J.: 1962, unpublished lecture given at Princeton University.

Faulkner, J.: 1974, this volume, p. 97; see also 1971, Astrophys. J. Letters 170, L99.

Field, G. F., Rees, M. J., and Sciama, D. W.: 1969, Comm. Astrophys. Space Sci. 1, 187.

Gibbons, G. W. and Hawking, S. W.: 1971, Phys. Rev. D4, 2191.

Goebel, C. J.: 1972, Astrophys. J. Letters 172, L95.

Hughes, H. G., III and Misner, C. W.: 1973, 'Gravitational Faraday Rotations of Photons and Gravitions 
Propagating in the Kerr Gravitational Field', University of Maryland, Center for Theoretical Physics Report, in preparation.

Lee, M. and Weber, J.: 1974, this volume, p. 35.

Madore, J.: 1973, Commun. Math. Phys. 30, 335.

Matzner, R. A. and Nutku, Y.: 1973, 'On the Method of Virtual Quanta and Gravitational Radiation', University of Maryland Center for Theoretical Physics Report 73-115, Proc. Roy. Soc. A, in press. Misner, C. W.: 1972a, Phys. Rev. Letters 28, 994.

Misner, C. W.: 1972b, Lecture at the Sixth Texas Conference on Relativistic Astrophysics, New York, N.Y., December 19, unpublished.

Misner, C. W.: 1974, article in forthcoming volume Ondes et radiations gravitationelles, Actes du Colloque International C.N.R.S. No. 220, Paris.

Misner, C. W., Breuer, R. A., Brill, D. R., Chrzanowski, P. L., Hughes, H. G., III, and Pereira, C. M.: 1972, Phys. Rev. Letters 28, 998.

Misner, C. W., Thorne, K. S., and Wheeler, J. A.: 1973, Gravitation, Freeman, San Francisco.

Press, W. H. and Thorne, K. S.: 1972, Ann. Rev. Astron. Astrophys. 10, 335.

Rees, M. J.: 1974, article in a forthcoming volume Ondes et radiations gravitationelles, Actes du Colloque International C.N.R.S. No. 220, Paris.

Regge, T. and Wheeler, J. A.: 1957, Phys. Rev. 108, 1063.

Ruffini, R. and Wheeler, J. A.: 1971, in A. F. Moore and V. Hardy (eds.), The Significance of Space Research for Fundamental Physics, ESRO SP-52, European Space Research Organization, Paris.

Sciama, D. W.: 1969, Nature 224, 1263.

Sciama, D.: 1972, Gen. Rel. Grav. 3, 149.

Tyson, J. A.: 1973, Physl Rev. Letters 31, 326.

Tyson, J. A. and Douglass, D. H.: 1972, Phys. Rev. Letters 28, 991.

Weber, J.: 1970, Phys. Rev. Letters 25, 180.

Weber, J., Lee, M., Gretz, D. J., Rydbeck, G., Trimble, V. L., and Steppel, S.: 1973, Phys. Rev. Letters 31, 779 .

Zerilli, F. J.: 1970, Phys. Rev. Letters 24, 737; see also Phys. Rev. D2, 2141. 